\title{
On the Number of Limit Cycles for Discontinuous Generalized Liénard Polynomial Differential Systems
}

\author{
Fangfang Jiang \\ Department of Mathematics, Tongji University, \\ Shanghai 200092, P. R. China \\ jiangfangfang87@126.com \\ Junping Shi \\ Department of Mathematics, College of William and Mary, \\ Williamsburg, VA 23187-8795, USA \\ shij@math.wm.edu \\ Jitao Sun* \\ Department of Mathematics, Tongji University, \\ Shanghai 200092, P. R. China \\ sunjt@sh163.net
}

Received November 12, 2014; Revised March 15, 2015

\begin{abstract}
In this paper, we investigate the number of limit cycles for a class of discontinuous planar differential systems with multiple sectors separated by many rays originating from the origin. In each sector, it is a smooth generalized Liénard polynomial differential system $x^{\prime}=-y+$ $g_{1}(x)+f_{1}(x) y$ and $y^{\prime}=x+g_{2}(x)+f_{2}(x) y$, where $f_{i}(x)$ and $g_{i}(x)$ for $i=1,2$ are polynomials of variable $x$ with any given degree. By the averaging theory of first-order for discontinuous differential systems, we provide the criteria on the maximum number of medium amplitude limit cycles for the discontinuous generalized Liénard polynomial differential systems. The upper bound for the number of medium amplitude limit cycles can be attained by specific examples.

Keywords: Discontinuous planar system; Liénard polynomial system; averaging theory; number of limit cycles.
\end{abstract}

\section{Introduction}

One of main topics of the qualitative theory of planar differential systems is the number and relative position of limit cycles. This problem restricted to continuous planar polynomial differential systems is the well known Hilbert's 16th problem, see for example [Li, 2003]. Up to now, there have been many achievements concerning the existence, uniqueness and the number of limit cycles, see for example [García et al., 2014; Justino \& Jorge, 2012;
Li \& Llibre, 2012; Llibre, 2010; Llibre \& Mereu, 2013, 2014; Llibre et al., 2015; Llibre \& Valls, 2012, 2013a, 2013b; Lloyd \& Lynch, 1988; Martins \& Mereu, 2014; Shen \& Han, 2013; Sun, 1992; Xiong \& Zhong, 2013] and references therein. A limit cycle bifurcating from a single degenerate singular point is called a small amplitude limit cycle, and the one bifurcating from periodic orbits of a linear center is called a medium amplitude limit cycle. In [Llibre \& Valls, 2012], the authors studied the number

*Author for correspondence 
of medium amplitude limit cycles for a class of polynomial differential systems of the form

$$
\begin{aligned}
& x^{\prime}=y-g_{1}(x)-f_{1}(x) y, \\
& y^{\prime}=-x-g_{2}(x)-f_{2}(x) y,
\end{aligned}
$$

where $f_{i}(x)$ and $g_{i}(x), i=1,2$, are polynomials of a given degree. For system (1), when $f_{1}(x)=$ $g_{1}(x)=0$, it is the generalized Liénard polynomial differential system. Note that the classical generalized Liénard polynomial differential equation is $x^{\prime \prime}+f(x) x^{\prime}+g(x)=0$, or equivalently

$$
x^{\prime}=y-F(x), \quad y^{\prime}=-g(x),
$$

where $F(x)=\int_{0}^{x} f(s) d s, f(x)$ and $g(x)$ are polynomials in the variable $x$. For system (2), when polynomials $F(x)$ and $g(x)$ have degrees $n$ and $m$ respectively, the generalization to discontinuous generalized Liénard polynomial differential systems was studied in [Llibre \& Mereu, 2013].

To the best of our knowledge, there are very few results concerning the number of limit cycles which bifurcate from a continuum of periodic orbits of differential systems perturbed by discontinuous Liénard polynomial differential systems. So we restrict our attention to a class of discontinuous and piecewise smooth planar polynomial differential systems with multiple sectors separated by rays starting from the origin.

In this paper, we investigate the maximum number of medium amplitude limit cycles for a discontinuous generalized Liénard polynomial differential system with even number $l$ sectors separated by $l / 2$ straight lines passing through the origin $(0,0)$, where the discontinuous set is of the form

$$
\bigcup_{k=0}^{l / 2-1}\left\{(x, y): y=\tan \left(\alpha+\frac{2 k \pi}{l}\right) x\right\} .
$$

We first consider the case where two different smooth generalized Liénard polynomial differential systems are defined alternatively in every two neighboring sectors on the plane. By applying the averaging theory of first-order for discontinuous differential systems, we obtain a lower bound for the maximum number of limit cycles and we show that the upper bound for the number of medium amplitude limit cycles can be attained by some constructed examples (see Example 4.1 for an example and Matlab numerical simulation). The obtained limit cycles bifurcate from the periodic orbits of the linear center $x^{\prime}=-y, y^{\prime}=x$. Moreover, we give the discussion on the result if there are different vector fields in different sectors respectively.

The paper is organized as follows. In the next section, we present some relevant preliminaries for the discontinuous generalized Liénard polynomial differential system. In Sec. 3, we first present the averaging theory of first-order for discontinuous differential systems, and then we prove our main results on the maximum number of medium amplitude limit cycles. In Sec. 4, we give two examples to illustrate the proved results. Conclusion is presented in Sec. 5.

\section{Preliminaries}

Consider the following discontinuous generalized Liénard polynomial differential system

$$
\begin{aligned}
& \left\{\begin{array}{l}
x^{\prime}=-y+\varepsilon\left(g_{11}(x)+f_{11}(x) y\right), \\
y^{\prime}=x+\varepsilon\left(g_{12}(x)+f_{12}(x) y\right), \quad h(x, y)>0,
\end{array}\right. \\
& \left\{\begin{array}{l}
x^{\prime}=-y+\varepsilon\left(g_{21}(x)+f_{21}(x) y\right), \\
y^{\prime}=x+\varepsilon\left(g_{22}(x)+f_{22}(x) y\right), \quad h(x, y)<0,
\end{array}\right.
\end{aligned}
$$

where $\varepsilon$ is a sufficiently small parameter, and $g_{i j}(x)$ and $f_{i j}(x)$ for $i, j=1,2$ are polynomials of variable $x$ with degrees $m$ and $n$ respectively. Let a function $h: \mathbb{R}^{2} \rightarrow \mathbb{R}$ be given by

$$
h(x, y)=\prod_{k=0}^{\frac{l}{2}-1}\left(y-\tan \left(\alpha+\frac{2 k \pi}{l}\right) x\right),
$$

with $l>1$ being an even integer and $\alpha \in \mathbb{R}$. Then

$$
h^{-1}(0)=\bigcup_{k=0}^{\frac{l}{2}-1}\left\{(x, y): y=\tan \left(\alpha+\frac{2 k \pi}{l}\right) x\right\}
$$

is a discontinuous set consisting of $l / 2$ straight lines passing through the origin $O(0,0)$, which divides the plane $\mathbb{R}^{2}$ into $l$ sectors with angle $2 \pi / l$.

When $\varepsilon=0$, the system (3) becomes a planar linear center as follows

$$
x^{\prime}=-y, \quad y^{\prime}=x .
$$

Note that the system (6) has a continuum of periodic orbits surrounding the origin and the orbits are counterclockwise oriented in the phase plane. In this paper, by using the averaging theory of first-order for discontinuous differential systems, we investigate the maximum number of medium amplitude limit cycles for the discontinuous generalized 
Liénard polynomial differential system (3) with $|\varepsilon|>0$ sufficiently small.

\section{Main Results}

First we recall the following result concerning the existence of limit cycles from the averaging theory of first-order for discontinuous differential systems.

Lemma 3.1 [Llibre \& Mereu, 2014]. Consider the following discontinuous differential equation

$$
x^{\prime}(t)=\varepsilon H(t, x)+\varepsilon^{2} G(t, x, \varepsilon),
$$

where $\varepsilon$ is a sufficiently small real number, functions $H(t, x), G(t, x, \varepsilon)$ are given by

$$
\begin{aligned}
H(t, x) & =H_{1}(t, x)+\operatorname{sgn}(h(t, x)) H_{2}(t, x), \\
G(t, x, \varepsilon) & =G_{1}(t, x, \varepsilon)+\operatorname{sgn}(h(t, x)) G_{2}(t, x, \varepsilon),
\end{aligned}
$$

functions $H_{1}, H_{2}: \mathbb{R} \times D \rightarrow \mathbb{R}^{n}, G_{1}, G_{2}: \mathbb{R} \times D \times$ $\mathbb{R} \rightarrow \mathbb{R}^{n}$ and $h: \mathbb{R} \times D \rightarrow \mathbb{R}$ are continuous, $T$ periodic in the variable $t$ and $D \subset \mathbb{R}^{n}$ is an open subset. Let $h$ be a $C^{1}$ function having 0 as a regular value. Denote by $\mathcal{M}=h^{-1}(0), \mathcal{N}=\{0\} \times D \nsubseteq \mathcal{M}$ and $\mathcal{K}=\mathcal{N} \backslash \mathcal{M} \neq \emptyset$. Moreover, define the averaging function $d: D \rightarrow \mathbb{R}^{n}$ of (7) to be

$$
d(x)=\int_{0}^{T} H(t, x) d t
$$

Assume that

(i) functions $H_{1}, H_{2}, G_{1}, G_{2}$ and $h$ are locally Lipschitz with respect to the variable $x$;

(ii) for $z_{0} \in \mathcal{K}$ with $d\left(z_{0}\right)=0$, there exists a neighborhood $V$ of $z_{0}$ such that $d(z) \neq 0$ for every $z \in \bar{V} \backslash\left\{z_{0}\right\}$ and $d_{B}\left(d, V, z_{0}\right) \neq 0$, where $d_{B}\left(d, V, z_{0}\right)$ denotes the Brouwer degree of the function $d$ with respect to the bounded open subset $V \subset D$ and the fixed point $z_{0}$;

(iii) if $\frac{\partial h}{\partial t}\left(t_{0}, z_{0}\right)=0$ for some $\left(t_{0}, z_{0}\right) \in \mathcal{M}$, then

$$
\left(\left[\nabla_{x} h, H_{1}\right]^{2}-\left[\nabla_{x} h, H_{2}\right]^{2}\right)\left(t_{0}, z_{0}\right)>0,
$$

where $[A, B]$ denotes the inner product of the vectors $A$ and $B, \nabla_{x} h$ denotes the gradient of $h(t, x)$ with respect to the variable $x$. Then for $0<|\varepsilon|$ sufficiently small, there exists a T-periodic solution $x(\cdot, \varepsilon)$ of $(7)$ such that $x(0, \varepsilon) \rightarrow z_{0}$ as $\varepsilon \rightarrow 0$.

Our main result of this paper is as follows.
Theorem 3.2. Consider the system (3) with $|\varepsilon|>$ 0 sufficiently small. Assume that the polynomials $g_{i j}(x)$ and $f_{i j}(x), i, j=1,2$ have degrees $m \geq 1$ and $n \geq 1$ respectively, then the maximum number of medium amplitude limit cycles bifurcating from the periodic orbits of system $(6)$ is $\lambda=\max \{m, n+1\}$.

Proof. Let $x=\rho \cos \theta, y=\rho \sin \theta$ with $\rho>0$, then the system (3) can be transformed into

$$
\begin{aligned}
\frac{d \rho}{d t}= & \varepsilon\left\{\cos \theta\left[g_{11}(\rho \cos \theta)+f_{11}(\rho \cos \theta) \rho \sin \theta\right]\right. \\
& \left.+\sin \theta\left[g_{12}(\rho \cos \theta)+f_{12}(\rho \cos \theta) \rho \sin \theta\right]\right\}, \\
\frac{d \theta}{d t}= & 1+\frac{\varepsilon}{\rho}\left\{\cos \theta\left[g_{12}(\rho \cos \theta)+f_{12}(\rho \cos \theta) \rho \sin \theta\right]\right. \\
& \left.-\sin \theta\left[g_{11}(\rho \cos \theta)+f_{11}(\rho \cos \theta) \rho \sin \theta\right]\right\},
\end{aligned}
$$

for $\theta \in\left(\alpha+\frac{4 k \pi}{l}, \alpha+\frac{(4 k+2) \pi}{l}\right), k=0,1, \ldots, \frac{l-2}{2}$ and

$$
\begin{aligned}
\frac{d \rho}{d t}= & \varepsilon\left\{\cos \theta\left[g_{21}(\rho \cos \theta)+f_{21}(\rho \cos \theta) \rho \sin \theta\right]\right. \\
& \left.+\sin \theta\left[g_{22}(\rho \cos \theta)+f_{22}(\rho \cos \theta) \rho \sin \theta\right]\right\}, \\
\frac{d \theta}{d t}= & 1+\frac{\varepsilon}{\rho}\left\{\cos \theta\left[g_{22}(\rho \cos \theta)+f_{22}(\rho \cos \theta) \rho \sin \theta\right]\right. \\
& \left.-\sin \theta\left[g_{21}(\rho \cos \theta)+f_{21}(\rho \cos \theta) \rho \sin \theta\right]\right\},
\end{aligned}
$$

for $\theta \in\left(\alpha+\frac{(4 k+2) \pi}{l}, \alpha+\frac{4(k+1) \pi}{l}\right), k=0,1, \ldots, \frac{l-2}{2}$. Denote by

$$
\begin{array}{ll}
g_{11}(x)=\sum_{i=0}^{m} a_{1, i} x^{i}, & g_{12}(x)=\sum_{i=0}^{m} b_{1, i} x^{i}, \\
g_{21}(x)=\sum_{i=0}^{m} a_{2, i} x^{i}, & g_{22}(x)=\sum_{i=0}^{m} b_{2, i} x^{i}, \\
f_{11}(x)=\sum_{i=0}^{n} c_{1, i} x^{i}, & f_{12}(x)=\sum_{i=0}^{n} d_{1, i} x^{i}, \\
f_{21}(x)=\sum_{i=0}^{n} c_{2, i} x^{i}, & f_{22}(x)=\sum_{i=0}^{n} d_{2, i} x^{i} .
\end{array}
$$

By taking the angle $\theta$ as the new independent variable and using Taylor series expansion, the system (3) becomes

$$
\begin{aligned}
\frac{d \rho}{d \theta}= & \varepsilon\left(\sum_{i=0}^{m} a_{1, i} \rho^{i} \cos ^{i+1} \theta+\sum_{i=0}^{n} c_{1, i} \rho^{i+1} \cos ^{i+1} \theta \sin \theta+\sum_{i=0}^{m} b_{1, i} \rho^{i} \cos ^{i} \theta \sin \theta+\sum_{i=0}^{n} d_{1, i} \rho^{i+1} \cos ^{i} \theta \sin ^{2} \theta\right) \\
& +\mathcal{O}\left(\varepsilon^{2}\right),
\end{aligned}
$$


for $\theta \in\left(\alpha+\frac{4 k \pi}{l}, \alpha+\frac{(4 k+2) \pi}{l}\right), k=0,1, \ldots, \frac{l-2}{2}$ and

$$
\begin{aligned}
\frac{d \rho}{d \theta}= & \varepsilon\left(\sum_{i=0}^{m} a_{2, i} \rho^{i} \cos ^{i+1} \theta+\sum_{i=0}^{n} c_{2, i} \rho^{i+1} \cos ^{i+1} \theta \sin \theta+\sum_{i=0}^{m} b_{2, i} \rho^{i} \cos ^{i} \theta \sin \theta+\sum_{i=0}^{n} d_{2, i} \rho^{i+1} \cos ^{i} \theta \sin ^{2} \theta\right) \\
& +\mathcal{O}\left(\varepsilon^{2}\right),
\end{aligned}
$$

for $\theta \in\left(\alpha+\frac{(4 k+2) \pi}{l}, \alpha+\frac{4(k+1) \pi}{l}\right), k=0,1, \ldots, \frac{l-2}{2}$. From (4) and (5) then

$$
\begin{aligned}
h^{-1}(0) & =\bigcup_{k=0}^{\frac{l}{2}-1}\left\{(\theta, \rho): \sin \theta=\cos \theta \tan \left(\alpha+\frac{2 k \pi}{l}\right)\right\}, \\
\frac{\partial h}{\partial \theta}(\theta, \rho) & =\sum_{p=0}^{\frac{l}{2}-1} \rho^{\frac{l}{2}}\left[\cos \theta+\sin \theta \tan \left(\alpha+\frac{2 p \pi}{l}\right)\right] \prod_{k \neq p}^{\frac{l}{2}-1}\left[\sin \theta-\cos \theta \tan \left(\alpha+\frac{2 k \pi}{l}\right)\right] .
\end{aligned}
$$

By some tedious but simple computations one has that $\frac{\partial h}{\partial \theta}(\theta, \rho) \neq 0$ for any $(\theta, \rho) \in h^{-1}(0)$.

On the other hand, for the purpose of the averaging function we need to use the following formulas [Gradshteyn \& Ryshik, 1994]

$$
\begin{aligned}
\int \cos ^{2 m} \theta d \theta & =\frac{1}{2^{2 m}}\left(\begin{array}{c}
2 m \\
m
\end{array}\right) \theta+\frac{1}{2^{2 m-1}} \sum_{j=0}^{m-1}\left(\begin{array}{c}
2 m \\
j
\end{array}\right) \frac{\sin (2 m-2 j) \theta}{2 m-2 j} \\
\int \cos ^{2 m+1} \theta d \theta & =\frac{1}{2^{2 m}} \sum_{j=0}^{m}\left(\begin{array}{c}
2 m+1 \\
j
\end{array}\right) \frac{\sin (2 m-2 j+1) \theta}{2 m-2 j+1} .
\end{aligned}
$$

Then the averaging function $d(\rho)$ of (8) and (9) is as follows

$$
d(\rho)=d_{1}(\rho)+d_{2}(\rho)+d_{3}(\rho)+d_{4}(\rho)
$$

where

$$
\begin{aligned}
& d_{1}(\rho)=\sum_{k=0}^{\frac{l-2}{2}} \sum_{i=0}^{m}\left(\int_{\alpha+\frac{4 k \pi}{l}}^{\alpha+\frac{(4 k+2) \pi}{l}} a_{1, i} \rho^{i} \cos ^{i+1} \theta d \theta+\int_{\alpha+\frac{(4 k+2) \pi}{l}}^{\alpha+\frac{4(k+1) \pi}{l}} a_{2, i} \rho^{i} \cos ^{i+1} \theta d \theta\right) \\
& d_{2}(\rho)=\sum_{k=0}^{\frac{l-2}{2}} \sum_{i=0}^{n}\left(\int_{\alpha+\frac{4 k \pi}{l}}^{\alpha+\frac{(4 k+2) \pi}{l}} c_{1, i} \rho^{i+1} \cos ^{i+1} \theta \sin \theta d \theta+\int_{\alpha+\frac{(4 k+2) \pi}{l}}^{\alpha+\frac{4(k+1) \pi}{l}} c_{2, i} \rho^{i+1} \cos ^{i+1} \theta \sin \theta d \theta\right), \\
& d_{3}(\rho)=\sum_{k=0}^{\frac{l-2}{2}} \sum_{i=0}^{m}\left(\int_{\alpha+\frac{4 k \pi}{l}}^{\alpha+\frac{(4 k+2) \pi}{l}} b_{1, i} \rho^{i} \cos ^{i} \theta \sin \theta d \theta+\int_{\alpha+\frac{(4 k+2) \pi}{l}}^{\alpha+\frac{4(k+1) \pi}{l}} b_{2, i} \rho^{i} \cos ^{i} \theta \sin \theta d \theta\right), \\
& d_{4}(\rho)=\sum_{k=0}^{\frac{l-2}{2}} \sum_{i=0}^{n}\left(\int_{\alpha+\frac{4 k \pi}{l}}^{\alpha+\frac{(4 k+2) \pi}{l}} d_{1, i} \rho^{i+1} \cos ^{i} \theta \sin ^{2} \theta d \theta+\int_{\alpha+\frac{(4 k+2) \pi}{l}}^{\alpha+\frac{4(k+1) \pi}{l}} d_{2, i} \rho^{i+1} \cos ^{i} \theta \sin ^{2} \theta d \theta\right) .
\end{aligned}
$$

Substituting (10) and (11) into the above equalities then

$$
d_{1}(\rho)=\sum_{k=0}^{\frac{l-2}{2}} \sum_{i=0}^{m} \frac{\rho^{i}}{2^{i}} \sum_{j=0}^{\left[\frac{i}{2}\right]}\left(\begin{array}{c}
i+1 \\
j
\end{array}\right)\left(a_{1, i} A_{i, j, k}+a_{2, i} \bar{A}_{i, j, k}\right)+\sum_{i=0}^{\left[\frac{m-1}{2}\right]}\left(a_{1,2 i+1}+a_{2,2 i+1}\right) \frac{\rho^{2 i+1}}{2^{2 i+2}}\left(\begin{array}{c}
2 i+2 \\
i+1
\end{array}\right) \frac{2 \pi}{l},
$$


with

$$
\begin{aligned}
A_{i, j, k} & =\frac{\sin \left[(i-2 j+1)\left(\alpha+\frac{(4 k+2) \pi}{l}\right)\right]-\sin \left[(i-2 j+1)\left(\alpha+\frac{4 k \pi}{l}\right)\right]}{i-2 j+1}, \\
\bar{A}_{i, j, k} & =\frac{\sin \left[(i-2 j+1)\left(\alpha+\frac{4(k+1) \pi}{l}\right)\right]-\sin \left[(i-2 j+1)\left(\alpha+\frac{(4 k+2) \pi}{l}\right)\right]}{i-2 j+1}, \\
d_{2}(\rho) & =-\sum_{k=0}^{\frac{l-2}{2}} \sum_{i=0}^{n}\left(c_{1, i} \rho^{i+1} B_{i, k}+c_{2, i} \rho^{i+1} \bar{B}_{i, k}\right) \\
d_{3}(\rho) & =-\sum_{k=0}^{\frac{l-2}{2}} \sum_{i=0}^{m}\left(b_{1, i} \rho^{i} C_{i, k}+b_{2, i} \rho^{i} \bar{C}_{i, k}\right)
\end{aligned}
$$

with

$$
\begin{aligned}
B_{i, k}= & \frac{\cos ^{i+2}\left(\alpha+\frac{(4 k+2) \pi}{l}\right)-\cos ^{i+2}\left(\alpha+\frac{4 k \pi}{l}\right)}{i+2}, \\
\bar{B}_{i, k}= & \frac{\cos ^{i+2}\left(\alpha+\frac{4(k+1) \pi}{l}\right)-\cos ^{i+2}\left(\alpha+\frac{(4 k+2) \pi}{l}\right)}{i+2}, \\
C_{i, k}= & \frac{\cos ^{i+1}\left(\alpha+\frac{(4 k+2) \pi}{l}\right)-\cos ^{i+1}\left(\alpha+\frac{4 k \pi}{l}\right)}{i+1}, \\
\bar{C}_{i, k}= & \frac{\cos ^{i+1}\left(\alpha+\frac{4(k+1) \pi}{l}\right)-\cos ^{i+1}\left(\alpha+\frac{(4 k+2) \pi}{l}\right)}{i+1}, \\
d_{4}(\rho)= & \sum_{i=0}^{\left[\frac{n}{2}\right]}\left(d_{1,2 i}+d_{2,2 i}\right) \rho^{2 i+1}\left[\frac{1}{2^{2 i}}\left(\begin{array}{c}
2 i \\
i
\end{array}\right)-\frac{1}{2^{2 i+2}}\left(\begin{array}{c}
2 i+2 \\
i+1
\end{array}\right)\right] \\
& +\sum_{k=0}^{\frac{l-2}{2}} \sum_{i=0}^{n} \frac{\rho^{i+1}}{2^{i-1}} \sum_{j=0}^{\left[\frac{i-1}{2}\right]}\left(\begin{array}{c}
i \\
j
\end{array}\right)\left(d_{1, i} D_{i, j, k}+d_{2, i} \bar{D}_{i, j, k}\right) \\
& -\sum_{k=0}^{\frac{l-2}{2}} \sum_{i=0}^{n} \frac{\rho^{i+1}}{2^{i+1}} \sum_{j=0}^{\left[\frac{i+1}{2}\right]}\left(\begin{array}{c}
i+2 \\
j
\end{array}\right)\left(d_{1, i} E_{i, j, k}+d_{2, i} \bar{E}_{i, j, k}\right),
\end{aligned}
$$

with

$$
D_{i, j, k}=\frac{\sin \left[(i-2 j)\left(\alpha+\frac{(4 k+2) \pi}{l}\right)\right]-\sin \left[(i-2 j)\left(\alpha+\frac{4 k \pi}{l}\right)\right]}{i-2 j}
$$


F. Jiang et al.

$$
\begin{aligned}
E_{i, j, k} & =\frac{\sin \left[(i-2 j+2)\left(\alpha+\frac{(4 k+2) \pi}{l}\right)\right]-\sin \left[(i-2 j+2)\left(\alpha+\frac{4 k \pi}{l}\right)\right]}{i-2 j+2}, \\
\bar{D}_{i, j, k} & =\frac{\sin \left[(i-2 j)\left(\alpha+\frac{4(k+1) \pi}{l}\right)\right]-\sin \left[(i-2 j)\left(\alpha+\frac{(4 k+2) \pi}{l}\right)\right]}{i-2 j}, \\
\bar{E}_{i, j, k} & =\frac{\sin \left[(i-2 j+2)\left(\alpha+\frac{4(k+1) \pi}{l}\right)\right]-\sin \left[(i-2 j+2)\left(\alpha+\frac{(4 k+2) \pi}{l}\right)\right]}{i-2 j+2} .
\end{aligned}
$$

Therefore, we obtain that

$$
\begin{aligned}
d(\rho)= & \sum_{i=0}^{\left[\frac{m-1}{2}\right]} \frac{\left(a_{1,2 i+1}+a_{2,2 i+1}\right) \rho^{2 i+1}}{2^{2 i+2}}\left(\begin{array}{c}
2 i+2 \\
i+1
\end{array}\right) \frac{2 \pi}{l}+\sum_{i=0}^{\left[\frac{n}{2}\right]} \frac{\left(d_{1,2 i}+d_{2,2 i}\right) \rho^{2 i+1}}{2^{2 i+1}(i+1)}\left(\begin{array}{c}
2 i \\
i
\end{array}\right) \frac{2 \pi}{l} \\
& +\sum_{k=0}^{\frac{l-2}{2}} \sum_{i=0}^{m} \frac{\rho^{i}}{2^{i}} \sum_{j=0}^{\left[\frac{i}{2}\right]}\left(\begin{array}{c}
i+1 \\
j
\end{array}\right)\left(a_{1, i} A_{i, j, k}+a_{2, i} \bar{A}_{i, j, k}\right)-\sum_{k=0}^{\frac{l-2}{2}} \sum_{i=0}^{n}\left(c_{1, i} B_{i, k}+c_{2, i} \bar{B}_{i, k}\right) \rho^{i+1} \\
& +\sum_{k=0}^{\frac{l-2}{2}} \sum_{i=0}^{n} \frac{\rho^{i+1}}{2^{i-1}} \sum_{j=0}^{\left[\frac{i-1}{2}\right]}\left(\begin{array}{c}
i \\
j
\end{array}\right)\left(d_{1, i} D_{i, j, k}+d_{2, i} \bar{D}_{i, j, k}\right)-\sum_{k=0}^{\frac{l-2}{2}} \sum_{i=0}^{m}\left(b_{1, i} C_{i, k}+b_{2, i} \bar{C}_{i, k}\right) \rho^{i} \\
& -\sum_{k=0}^{\frac{l-2}{2}} \sum_{i=0}^{n} \frac{\rho^{i+1}}{2^{i+1}} \sum_{j=0}^{\left[\frac{i+1}{2}\right]}\left(\begin{array}{c}
i+2 \\
j
\end{array}\right)\left(d_{1, i} E_{i, j, k}+d_{2, i} \bar{E}_{i, j, k}\right) .
\end{aligned}
$$

From the above equality we observe that the function $d(\rho)$ is a polynomial of variable $\rho$ with degree $\max \{m, n+1\}$, so the polynomial $d(\rho)$ has at most $\lambda=\max \{m, n+1\}$ positive roots, that is, the maximum number of bifurcating medium amplitude limit cycles is $\lambda$. Moreover, if there exists some $\rho_{0}>0$ such that $d\left(\rho_{0}\right)=0$ and $d^{\prime}\left(\rho_{0}\right) \neq 0$ then the Brouwer degree $d_{B}\left(d, V, \rho_{0}\right) \neq 0$ for some small open neighborhood $V$ of $\rho_{0}$. Therefore, by Lemma 3.1 for $0<|\varepsilon|$ sufficiently small the discontinuous system (3) has at most $\lambda$ medium amplitude limit cycles bifurcating from the periodic orbits of the linear center $x^{\prime}=-y, y^{\prime}=x$. This completes the proof of Theorem 3.2.

Remark 3.3. It should be noted that for some given degree $m \geq 1$ and $n \geq 1$, there exist discontinuous generalized Liénard polynomial differential systems having exactly $\lambda=\max \{m, n+1\}$ medium amplitude limit cycles, see Example 4.1 for an example with $m=n=1$.

When $\alpha=0$ and $l=2$, we have

$$
A_{i, j, k}=\bar{A}_{i, j, k}=0, \quad D_{i, j, k}=\bar{D}_{i, j, k}=0
$$

$$
\text { and } \quad E_{i, j, k}=\bar{E}_{i, j, k}=0 \text {. }
$$

In this case, the function $h: \mathbb{R}^{2} \rightarrow \mathbb{R}$ is of the form $h(x, y)=y$, and the discontinuous generalized Liénard polynomial differential system with two zones separated by the straight line $\{y=0\}$ is as follows

$$
\begin{aligned}
& \left\{\begin{array}{l}
x^{\prime}=-y+\varepsilon\left(g_{11}(x)+f_{11}(x) y\right), \\
y^{\prime}=x+\varepsilon\left(g_{12}(x)+f_{12}(x) y\right), \quad y>0,
\end{array}\right. \\
& \left\{\begin{array}{l}
x^{\prime}=-y+\varepsilon\left(g_{21}(x)+f_{21}(x) y\right), \\
y^{\prime}=x+\varepsilon\left(g_{22}(x)+f_{22}(x) y\right), \quad y<0 .
\end{array}\right.
\end{aligned}
$$

We have the following result:

Corollary 3.4. Consider the system (12) with $0<$ $|\varepsilon|$ sufficiently small, then the maximum number of medium amplitude limit cycles is

$$
\lambda=\max \{m, n+1\} .
$$

Proof. In the same way as Theorem 3.2 then the averaging function $d(\rho)$ is of the form 


$$
\begin{aligned}
d(\rho)= & \int_{0}^{\pi}\left(\sum_{i=0}^{m} a_{1, i} \rho^{i} \cos ^{i+1} \theta+\sum_{i=0}^{n} c_{1, i} \rho^{i+1} \cos ^{i+1} \theta \sin \theta+\sum_{i=0}^{m} b_{1, i} \rho^{i} \cos ^{i} \theta \sin \theta\right. \\
& \left.+\sum_{i=0}^{n} d_{1, i} \rho^{i+1} \cos ^{i} \theta \sin ^{2} \theta\right) d \theta+\int_{\pi}^{2 \pi}\left(\sum_{i=0}^{m} a_{2, i} \rho^{i} \cos ^{i+1} \theta+\sum_{i=0}^{n} c_{2, i} \rho^{i+1} \cos ^{i+1} \theta \sin \theta\right. \\
& \left.+\sum_{i=0}^{m} b_{2, i} \rho^{i} \cos ^{i} \theta \sin \theta+\sum_{i=0}^{n} d_{2, i} \rho^{i+1} \cos ^{i} \theta \sin ^{2} \theta\right) d \theta \\
= & \sum_{i=0}^{\left[\frac{m-1}{2}\right]}\left(a_{1,2 i+1}+a_{2,2 i+1}\right) \rho^{2 i+1} \frac{\pi(2 i+1) ! !}{(2 i+2) ! !}+\sum_{i=0}^{\left[\frac{n-1}{2}\right]}\left(c_{1,2 i+1}-c_{2,2 i+1}\right) \rho^{2 i+2} \frac{2}{2 i+3} \\
& +\sum_{i=0}^{\left[\frac{m}{2}\right]}\left(b_{1,2 i}-b_{2,2 i}\right) \rho^{2 i} \frac{2}{2 i+1}+\sum_{i=0}^{\left[\frac{n}{2}\right]}\left(d_{1,2 i}+d_{2,2 i}\right) \rho^{2 i+1} \frac{\pi(2 i-1) ! !}{(2 i+2) ! !}
\end{aligned}
$$

Therefore, we obtain that for $|\varepsilon|>0$ sufficiently small the discontinuous system (12) has at most $\lambda=\max \{m, n+1\}$ that bifurcate medium amplitude limit cycles and then the conclusion holds.

When there are different vector fields in the different sectors respectively, we consider the following discontinuous generalized Liénard polynomial differential system of the form

$$
\left\{\begin{array}{l}
x^{\prime}=-y+\varepsilon\left(g_{i 1}(x)+f_{i 1}(x) y\right), \\
y^{\prime}=x+\varepsilon\left(g_{i 2}(x)+f_{i 2}(x) y\right), \quad(x, y) \in S_{i},
\end{array}\right.
$$

where $S_{i}$ denotes the $i$ th sector separated by $l / 2$ straight lines passing through the origin, $l$ is a positive even integer, and the polynomials $g_{i j}(x)$ and $f_{i j}(x)$ for $i=1,2, \ldots, l, j=1,2$ are

$$
\begin{array}{ll}
g_{i 1}(x)=\sum_{j=0}^{m} a_{i, j} x^{j}, & g_{i 2}(x)=\sum_{j=0}^{m} b_{i, j} x^{j}, \\
f_{i 1}(x)=\sum_{j=0}^{n} c_{i, j} x^{j}, & f_{i 2}(x)=\sum_{j=0}^{n} d_{i, j} x^{j},
\end{array}
$$

satisfying $m \geq 1, n \geq 1$. Then we have the following result.

Theorem 3.5. Consider the system (13) with $0<$ $|\varepsilon|$ sufficiently small, then the maximum number of medium amplitude limit cycles bifurcating from the periodic orbits of $(6)$ is $\lambda=\max \{m, n+1\}$.

Proof. Let $x=\rho \cos \theta, y=\rho \sin \theta$ with $\rho>0$, then the system (13) is transformed into

$$
\begin{aligned}
\frac{d \rho}{d \theta}= & \varepsilon\left(\sum_{j=0}^{m} a_{i, j} \rho^{j} \cos ^{j+1} \theta\right. \\
& +\sum_{j=0}^{n} c_{i, j} \rho^{j+1} \cos ^{j+1} \theta \sin \theta \\
& +\sum_{j=0}^{m} b_{i, j} \rho^{j} \cos ^{j} \theta \sin \theta \\
& \left.+\sum_{j=0}^{n} d_{i, j} \rho^{j+1} \cos ^{j} \theta \sin ^{2} \theta\right)+\mathcal{O}\left(\varepsilon^{2}\right)
\end{aligned}
$$

for $\theta \in\left(\alpha+\frac{(2 i-2) \pi}{l}, \alpha+\frac{2 i \pi}{l}\right), i=1,2, \ldots, l$. With the same proof as Theorem 3.2, it follows from Lemma 3.1 that the conclusion holds.

Remark 3.6. For system (13) with (14), if $S_{i}, i=1$, $2, \ldots, l$ is the $i$ th sector separated by $l$ rays starting from the origin then the plane $\mathbb{R}^{2}$ is divided by the $l$ discontinuous rays into $l$ sectors. In this case, we have the same conclusion as Theorem 3.5.

Remark 3.7. Note that the maximum number of medium amplitude limit cycles is independent of the number of sectors, but it depends on the degree of the polynomials $g_{i j}(x)$ and $f_{i j}(x)$.

\section{Examples}

Example 4.1. Consider the following discontinuous generalized Liénard polynomial differential system with two zones separated by the straight line 
$\{y=0\}:$

$$
\begin{aligned}
& \left\{\begin{array}{l}
x^{\prime}=-y+\varepsilon\left(\frac{2}{\pi} x-\frac{3}{2} x y\right), \\
y^{\prime}=x+\varepsilon(x-1), \quad y>0,
\end{array}\right. \\
& \left\{\begin{array}{l}
x^{\prime}=-y+\varepsilon\left(\frac{2}{\pi} x+1+y\right), \\
y^{\prime}=x+\varepsilon\left(x+\frac{2}{\pi} y+x y\right), \quad y<0 .
\end{array}\right.
\end{aligned}
$$

From the system (15), we observe that

$$
\begin{aligned}
& g_{11}(x)=\frac{2}{\pi} x, \quad g_{12}(x)=x-1, \\
& g_{21}(x)=\frac{2}{\pi} x+1, \quad g_{22}(x)=x, \\
& f_{11}(x)=-\frac{3}{2} x, \quad f_{12}(x)=0, \\
& f_{21}(x)=1, \quad f_{22}(x)=\frac{2}{\pi}+x
\end{aligned}
$$

so the functions $g_{i j}(x), f_{i j}(x)$ for $i, j=1,2$ have degrees $m=1$ and $n=1$, respectively.

By Lemma 3.1, the averaging function $d(\rho)$ of $(15)$ is as follows

$$
\begin{aligned}
d(\rho)= & \int_{0}^{\pi}\left[\cos \theta\left(\frac{2}{\pi} \rho \cos \theta-\frac{3}{2} \rho^{2} \sin \theta \cos \theta\right)\right. \\
& +\sin \theta(\rho \cos \theta-1)] d \theta \\
& +\int_{\pi}^{2 \pi}\left[\cos \theta\left(\frac{2}{\pi} \rho \cos \theta+1+\rho \sin \theta\right)\right. \\
& +\sin \theta\left(\rho \cos \theta+\frac{2}{\pi} \rho \sin \theta\right. \\
& \left.\left.+\rho^{2} \sin \theta \cos \theta\right)\right] d \theta \\
= & -\rho^{2}+3 \rho-2=-(\rho-2)(\rho-1) .
\end{aligned}
$$

It is easy to see that the zeros of $d(\rho)$ are $\rho_{1}=2$ and $\rho_{2}=1$ satisfying $d^{\prime}(1)>0$ and $d^{\prime}(2)<0$.

Therefore, by Theorem 3.2 we obtain that for $|\varepsilon|>0$ sufficiently small, the discontinuous system (15) has two medium amplitude limit cycles. One is a stable limit cycle bifurcating from the periodic orbit of radius $\rho_{1}=2$ of the linear center $x^{\prime}=-y, y^{\prime}=x$, and the other is an unstable limit cycle bifurcating from the periodic orbit of radius $\rho_{2}=1$. By Matlab we see that the discontinuous

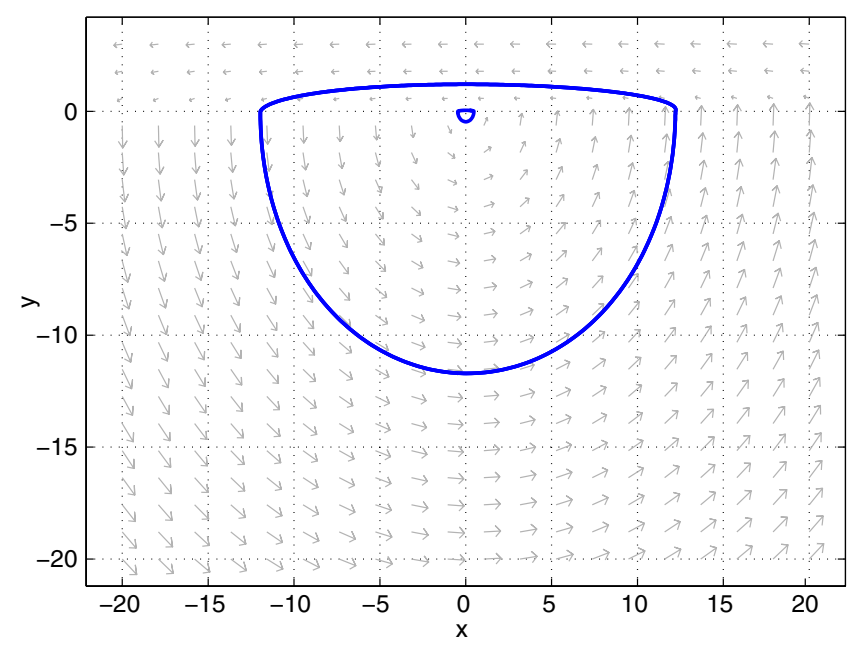

Fig. 1. Two medium amplitude limit cycles of (15) with $\varepsilon=0.01$.

system (15) has indeed two medium amplitude limit cycles (see Fig. 1), the bigger one is a stable limit cycle and the smaller one is an unstable limit cycle.

Example 4.2. Let $l=4$ and $\alpha=\frac{\pi}{4}$, and we consider the following discontinuous generalized Liénard polynomial differential system

$$
\begin{aligned}
& \left\{\begin{array}{l}
x^{\prime}=-y+\varepsilon\left(2 x^{2}+x y\right), \\
y^{\prime}=x+\varepsilon\left(1+x+x^{2}+\frac{8}{\pi} x^{2} y\right), \quad h(x, y)>0,
\end{array}\right. \\
& \left\{\begin{array}{l}
x^{\prime}=-y+\varepsilon\left(1+3 x^{2}-\frac{4 x}{\pi+2}+y+x^{2} y\right), \\
y^{\prime}=x+\varepsilon\left(x^{2}+x y\right), \quad h(x, y)<0 .
\end{array}\right.
\end{aligned}
$$

It follows that the scalar function $h: \mathbb{R}^{2} \rightarrow \mathbb{R}$ is defined as $h(x, y)=(y-x)(y+x)$ and the discontinuity set is of the form $h^{-1}(0)=\{(x, y): y=$ $x\} \cup\{(x, y): y=-x\}$. Obviously,

$$
\begin{aligned}
& g_{11}(x)=2 x^{2}, \quad g_{12}(x)=1+x+x^{2}, \\
& g_{21}(x)=1+3 x^{2}-\frac{4 x}{\pi+2}, \quad g_{22}(x)=x^{2}, \\
& f_{11}(x)=x, \quad f_{12}(x)=\frac{8}{\pi} x^{2}, \\
& f_{21}(x)=x^{2}+1, \quad f_{22}(x)=x
\end{aligned}
$$

then the polynomials $g_{i j}(x), f_{i j}(x)$ for $i, j=1,2$, have degrees $m=2$ and $n=2$ respectively. 
By Lemma 3.1, the averaging function $d(\rho)$ of $(16)$ is as follows

$$
\begin{aligned}
d(\rho)= & \int_{\frac{\pi}{4}}^{\frac{3 \pi}{4}}\left[\cos \theta\left(g_{11}(\rho \cos \theta)+f_{11}(\rho \cos \theta) \rho \sin \theta\right)+\sin \theta\left(g_{12}(\rho \cos \theta)+f_{12}(\rho \cos \theta) \rho \sin \theta\right)\right] d \theta \\
& +\int_{\frac{3 \pi}{4}}^{\frac{5 \pi}{4}}\left[\cos \theta\left(g_{21}(\rho \cos \theta)+f_{21}(\rho \cos \theta) \rho \sin \theta\right)+\sin \theta\left(g_{22}(\rho \cos \theta)+f_{22}(\rho \cos \theta) \rho \sin \theta\right)\right] d \theta \\
& +\int_{\frac{5 \pi}{4}}^{\frac{7 \pi}{4}}\left[\cos \theta\left(g_{11}(\rho \cos \theta)+f_{11}(\rho \cos \theta) \rho \sin \theta\right)+\sin \theta\left(g_{12}(\rho \cos \theta)+f_{12}(\rho \cos \theta) \rho \sin \theta\right)\right] d \theta \\
& +\int_{\frac{7 \pi}{4}}^{\frac{9 \pi}{4}}\left[\cos \theta\left(g_{21}(\rho \cos \theta)+f_{21}(\rho \cos \theta) \rho \sin \theta\right)+\sin \theta\left(g_{22}(\rho \cos \theta)+f_{22}(\rho \cos \theta) \rho \sin \theta\right)\right] d \theta \\
= & -\rho^{3}+3 \rho^{2}-2 \rho=-\rho(\rho-2)(\rho-1) .
\end{aligned}
$$

Therefore, for $|\varepsilon|>0$ sufficiently small the discontinuous system (16) has two medium amplitude limit cycles. One is bifurcating from the periodic orbit of radius 2 centered at the origin and it is stable. The other is bifurcating from the periodic orbit of radius 1 and it is unstable.

Remark 4.3. Note that for smooth generalized Liénard polynomial differential system $x^{\prime}=-y+$ $\varepsilon\left(g_{1}(x)+f_{1}(x) y\right), y^{\prime}=x+\varepsilon\left(g_{2}(x)+f_{2}(x) y\right)$, where $f_{i}(x)$ and $g_{i}(x), i=1,2$ are polynomials with degrees $n$ and $m$, respectively. Then the maximum number of medium amplitude limit cycles by using the averaging theory of first-order is $\max \left\{\left[\frac{m-1}{2}\right],\left[\frac{n}{2}\right]\right\}$. So roughly speaking, Theorem 3.2 in this paper shows that the discontinuous generalized Liénard polynomial differential systems can have at least one more limit cycle than the corresponding smooth ones, see for instance Examples 4.1 and 4.2 .

\section{Conclusion}

In this paper, we have investigated the maximum number of medium amplitude limit cycles for a class of discontinuous generalized Liénard polynomial differential systems with $l$ sectors separated by $l / 2$ straight lines passing through the origin of coordinates. The discontinuous system consists of two different smooth generalized Liénard polynomial differential systems of the form $x^{\prime}=-y+$ $g_{1}(x)+f_{1}(x) y, y^{\prime}=x+g_{2}(x)+f_{2}(x) y$, located alternatively in every two neighboring sectors, where the polynomials $f_{i}(x)$ and $g_{i}(x)$ for $i=1,2$ have degrees $n$ and $m$ respectively. By using the averaging theory of first-order for discontinuous differential systems, we have obtained that for $|\varepsilon|>$ 0 sufficiently small, the discontinuous generalized Liénard polynomial differential system has at most $\max \{m, n+1\}$ medium amplitude limit cycles and the upper bound $\max \{m, n+1\}$ of the number of medium amplitude limit cycles can be attained for some values $m$ and $n$. Moreover, we have also obtained the same result if there are different vector fields in different sectors, respectively.

\section{Acknowledgments}

The authors are grateful to the referee for his/her constructive comments. This work was revised when the first author visited College of William and Mary in Spring 2015. She expresses her gratitude to Department of Mathematics, College of William and Mary for warm hospitality, and is also very grateful to Professor Shi for valuable guidance.

This work is supported by the NSF of China under grant 61174039 .

\section{References}

García, B., Llibre, J. \& Pérez del Río, J. S. [2014] "Limit cycles of generalized Liénard polynomial differential systems via averaging theory," Chaos Solit. Fract. 6263, 1-9.

Gradshteyn, I. S. \& Ryshik, I. M. [1994] Table of Integrals, Series and Products, 5th edition, ed. Jeffrey, A. (Academic Press, NY).

Justino, A. R. \& Jorge, L. L. [2012] "On the maximum number of limit cycles of a class of generalized Liénard differential systems," Int. J. Bifurcation and Chaos 22, 1-14. 
Li, J. B. [2003] "Hilbert's 16th problem and bifurcations of planar polynomial vector fields," Int. J. Bifurcation and Chaos 13, 47-106.

Li, C. Z. \& Llibre, J. [2012] "Uniqueness of limit cycles for Liénard differential equations of degree four," J. Diff. Eqs. 252, 3142-3162.

Llibre, J. [2010] "The number of limit cycles in a Z(2)equivariant Liénard system," Math. Proc. Camb. Phil. Soc. 148, 363-383.

Llibre, J. \& Valls, C. [2012] "On the number of limit cycles of a class of polynomial differential systems," Proc. Roy. Soc. A 468, 2347-2360.

Llibre, J. \& Mereu, A. C. [2013] "Limit cycles for discontinuous generalized Liénard polynomial differential equations," Electron. J. Diff. Eqs. 2013, 1-8.

Llibre, J. \& Valls, C. [2013a] "On the number of limit cycles for a generalization of Liénard polynomial differential systems," Int. J. Bifurcation and Chaos 23, $1-16$

Llibre, J. \& Valls, C. [2013b] "Limit cycles for a generalization of polynomial Liénard differential systems," Chaos Solit. Fract. 46, 65-74.
Llibre, J. \& Mereu, A. C. [2014] "Limit cycles for discontinuous quadratic differential systems with two zones," J. Math. Anal. Appl. 413, 763-775.

Llibre, J., Novaes, D. D. \& Teixeira, M. A. [2015] "On the birth of limit cycles for non-smooth dynamical systems," Bull. Sci. Math. 139, 229-244.

Lloyd, N. D. \& Lynch, S. [1988] "Small-amplitude limit cycles of certain Liénard systems," Proc. Roy. Soc. Lond. A 418, 199-208.

Martins, R. M. \& Mereu, A. C. [2014] "Limit cycles in discontinuous classical Liénard equations," Nonlin. Anal: Real World Appl. 20, 67-73.

Shen, J. H. \& Han, M. A. [2013] "Bifurcations of canard limit cycles in several singularly perturbed generalized polynomial Liénard systems," Discr. Contin. Dyn. Syst. Ser. A 33, 3085-3108.

Sun, J. T. [1992] "Boundedness of solutions of Liénard systems and existence of limit cycles," Gaoxiao Yingyong Shuxue Xuebao 7, 184-191.

Xiong, Y. Q. \& Zhong, H. [2013] "The number of limit cycles in a Z(2)-equivariant Liénard system," Int. J. Bifurcation and Chaos 23, 1-17. 